\title{
A Wireless PDA-Based Physiological Monitoring System for Patient Transport
}

\author{
Yuan-Hsiang Lin, I-Chien Jan, Patrick Chow-In Ko, Yen-Yu Chen, Jau-Min Wong, and Gwo-Jen Jan
}

\begin{abstract}
This paper proposes a mobile patient monitoring system, which integrates current personal digital assistant (PDA) technology and wireless local area network (WLAN) technology. At the patient's location, a wireless PDA-based monitor is used to acquire continuously the patient's vital signs, including heart rate, three-lead electrocardiography, and $\mathrm{SpO}_{2}$. Through the WLAN, the patient's biosignals can be transmitted in real-time to a remote central management unit, and authorized medical staffs can access the data and the case history of the patient, either by the central management unit or the wireless devices. A prototype of this system has been developed and implemented. The system has been evaluated by technical verification, clinical test, and user survey. The evaluation of performance yields a high degree of satisfaction (mean $=4.64$, standard deviation- $\mathrm{SD}=0.53$ in a five-point Likert scale) of users who used the PDA-based system for intrahospital transport. The results also show that the wireless PDA model is superior to the currently used monitors both in mobility and in usability, and is, therefore, better suited to patient transport.
\end{abstract}

Index Terms-Mobile vital signs monitor, patient transport, personal digital assistant (PDA), physiological monitoring, wireless local area network (WLAN), wireless patient monitoring, wireless telemedicine.

\section{INTRODUCTION}

$\mathbf{T}$ ODAY, MORE and more intrahospital transport of patients is required in order to perform special examination or therapy [1]. The key to success of all critical care transport is the continuous monitoring of vital signs including single-strip electrocardiography (ECG), oxygen saturation by pulse oximetry $\left(\mathrm{SpO}_{2}\right)$, heart rate $(\mathrm{HR})$, and blood pressure [2]. Knowing the physiological changes of patients while they are being transported supports a preventive and early treatment strategy that makes transport safe and smooth. In cases of intrahospital or interhospital transport, patients are generally confined in a small carrier space such as an ambulance, a stretcher, or a wheelchair. In our experience, health-care personnel are often confronted by several inconveniences associated with the use

Manuscript received August 29, 2003; revised April 20, 2004. This work was supported in part by the National Taiwan University Hospital under Research Grant NTUH92N007.

Y.-H. Lin, I-C. Jan, and Y.-Y. Chen are with the Department of Electrical Engineering, National Taiwan University, Taipei 10617, Taiwan, R.O.C. (e-mail: f86921008@ntu.edu.tw; f86008@mail.ee.ntu.edu.tw).

P. C.-I. Ko is with the Department of Emergency Medicine, National Taiwan University Hospital, Taipei 100, Taiwan, R.O.C.

J.-M. Wong is with the Institute of Biomedical Engineering, National Taiwan University, Taipei 100, Taiwan, R.O.C., and also with the Department of Internal Medicine, National Taiwan University Hospital, Taipei 100, Taiwan, R.O.C.

G.-J. Jan is with the Department of Electrical Engineering and Graduate Institute of Electro-Optical Engineering, National Taiwan University, Taipei 10617, Taiwan, R.O.C. (e-mail: gjjan@cc.ee.ntu.edu.tw).

Digital Object Identifier 10.1109/TITB.2004.837829 of monitoring equipment during transport. First, the bulkiness of the monitor makes it difficult to lift and transport. Second, since the monitors are usually put on a trolley or at the end of the stretcher and the leads between the patient and the equipment are frequently wrapped around intravenous lines or other tubes, it can easily lead to disconnection during transport. Moreover, when patients receive radiographic examination, the monitors must be placed beside them during radiation process, and health-care personnel cannot remain in the control room to monitor the physiological data on the monitor screen.

Recently, the fast development of mobile technologies, including increased communication bandwidth and miniaturization of mobile terminals, has accelerated developments in the field of mobile telemedicine [3]. Wireless patient monitoring systems not only increase the mobility of patients and medical personnel but also improve the quality of health care [4]. With respect to the remote monitoring of patients, many groups have demonstrated the transmission of vital biosignals using global system for mobile communication (GSM) technology [5], [6]. Some researchers have used cellular phones to transmit vital signs from the ambulance to the hospital, either in store-and-forward mode [7] or in real-time mode [8].

However, few attempts have so far been made to telemonitor patients continuously during intrahospital transport. Transfers from one location within a hospital to another are sometimes confronted with temporary loss of the current state of patients' condition, a situation which can readily be alleviated via the use of a wireless device [9]. In this paper, we developed a wireless patient monitoring system which integrates current personal digital assistant (PDA) [10] and wireless local area network (WLAN) [11] technology, which also enables continuous monitoring during intrahospital patient transport. The PDA-based monitoring system has been preliminary evaluated and compared with the present system, from the perspective of its operators.

\section{METHODS}

\section{A. Overview of the System}

The aim of this study is to design and implement a mobile system for monitoring vital signs, and to facilitate the continuous monitoring of patients during transport. Fig. 1 shows the architecture of the proposed system. The telemedicine system consists mainly of two parts-1) the mobile unit, which is set up around the patient to acquire the patient's physiological data, and 2) the management unit, which enables the medical staffs 


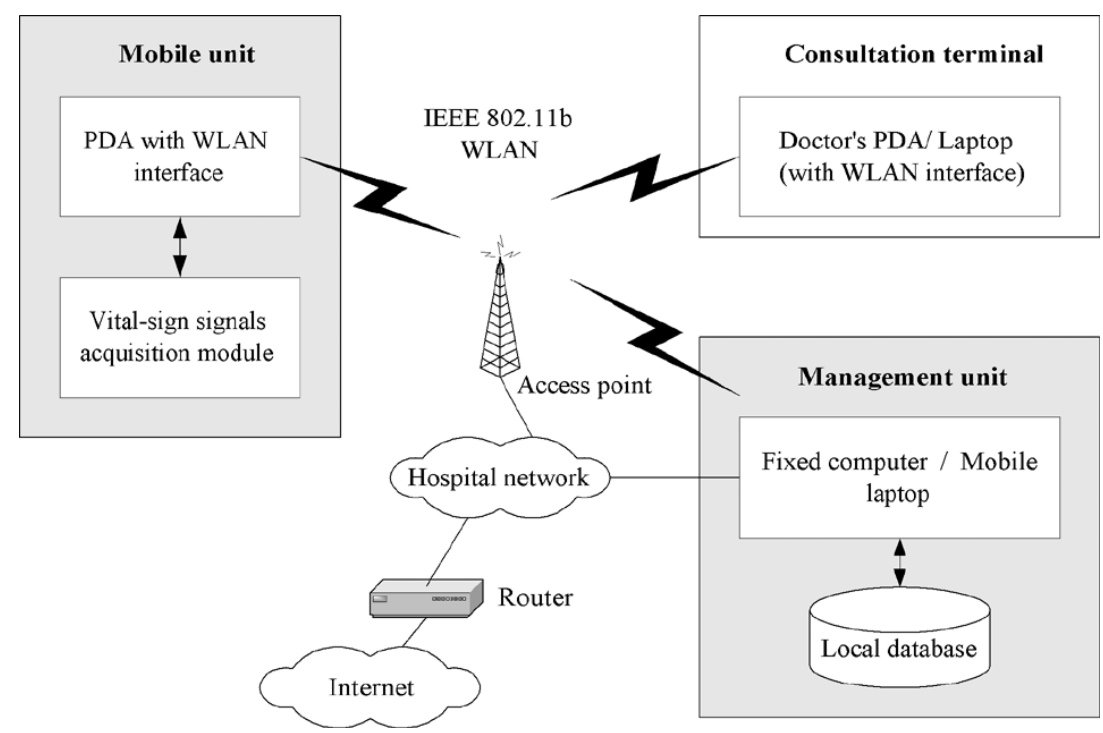

Fig. 1. Architecture of the wireless telemedicine system.

to telemonitor the patient's condition in real-time. The management unit is from either a fixed computer within an existing hospital network or a mobile laptop via WLAN.

The major design requirements of the mobile unit are as follows: 1) it should be portable and lightweight, which means easy to carry; 2) it should have power autonomy of more than 60 min to support patient transport; 3) it should have a userfriendly interface; 4) it should collect and display critical biosignals, including three-lead ECG, $\mathrm{HR}$, and $\mathrm{SpO}_{2}$; 5) it should record patient information and data; and 6) it should support wireless communication. On the other hand, the design requirements of the management unit are as follows: 1) it must have an easy-to-use interface; 2) it must display critical biosignals and analysis of data; 3 ) it must record, retrieve, and manage patient information and data (local database); and 4) it must be connectable to the Internet to transmit data and distribute information.

Furthermore, at the consultation terminals such as wireless PDAs or laptops, the medical staffs can use them either to monitor the physiological parameters and waveforms of a remote patient online or to access his or her case history through the wireless connection to the management unit.

Wireless connection in the studied hospital has been established by WLAN technology (IEEE 802.11b) [12] with speeds up to $11 \mathrm{Mb} / \mathrm{s}$. An access point acts as a wireless bridge for the network data to be transmitted to and received from the existing wired hospital network. With multiple access points linked to a wired network, it allows efficient sharing of network resources throughout an entire building. The distance set between each access point was less than $30 \mathrm{~m}$ because of the radius of indoor coverage for typical WLAN and regional geography limitation. Devices with WLAN interface can roam among the access points.

The transmission of data between a mobile unit and a management unit is implemented by the client server architecture. In the proposed design, the mobile unit serves as the client end and the management unit serves as the server end. Communication depends on the transmission control/Internet protocol for error-free medical data transmission. A specific service set identifier (SSID) and the 40-bit wired equivalent privacy (WEP) model of the IEEE $802.11 \mathrm{~b}$ are used to protect data during transmission. All users are required to enter a user name and password to the system via a remote authentication dial-in user service server. In addition, a robust advanced encryption standard (AES) algorithm [13] is implemented in the designed C++ program, permitting both mobile unit and management unit to perform end-to-end encryption.

\section{B. Mobile Unit}

The mobile unit in this study is comprised of a designed vital-sign signals acquisition module and a Pocket PC (HP iPAQ H5450). Multiple vital-sign parameters, which include the three-lead ECG, $\mathrm{SpO}_{2}$, and $\mathrm{HR}$, can be measured by this unit. Fig. 2 shows the design architecture of this mobile unit. This signals acquisition module acquires the three-lead ECG and dual-wavelength photoplethysmographic (PPG) signals, and converts them into digital data. Through an RS232 connection, the Pocket PC receives the physiological data and computes the $\mathrm{SpO}_{2}$ and HR parameters. According to user commands, the mobile unit can display waveforms in real-time, store data locally, and trigger an alarm. With regard to remote monitoring, the Pocket PC transfers these physiological data to a remote management unit in real-time by its built-in WLAN device.

1) Module for Acquiring Vital-Sign Signals: Fig. 3 shows the diagram of the designed vital-sign signals acquisition module. The vital-sign signals acquisition module consists of ECG signal conditioning circuits, pulse oximeter analog circuits, and a microcontroller. This module is powered by four rechargeable AA batteries and is packaged as a jacket of the Pocket PC. The core control unit of the module is an 8-bit microcontroller, PIC16F877, which has an on-chip eight-channel 10-bit analog-to-digital converter (ADC). The three-lead ECG signals were amplified with a gain of 700 , filtered $(0.5-50 \mathrm{~Hz})$, and then fed into the inputs of the ADC in the microcontroller. The pulse oximeter analog circuits were designed based on the 


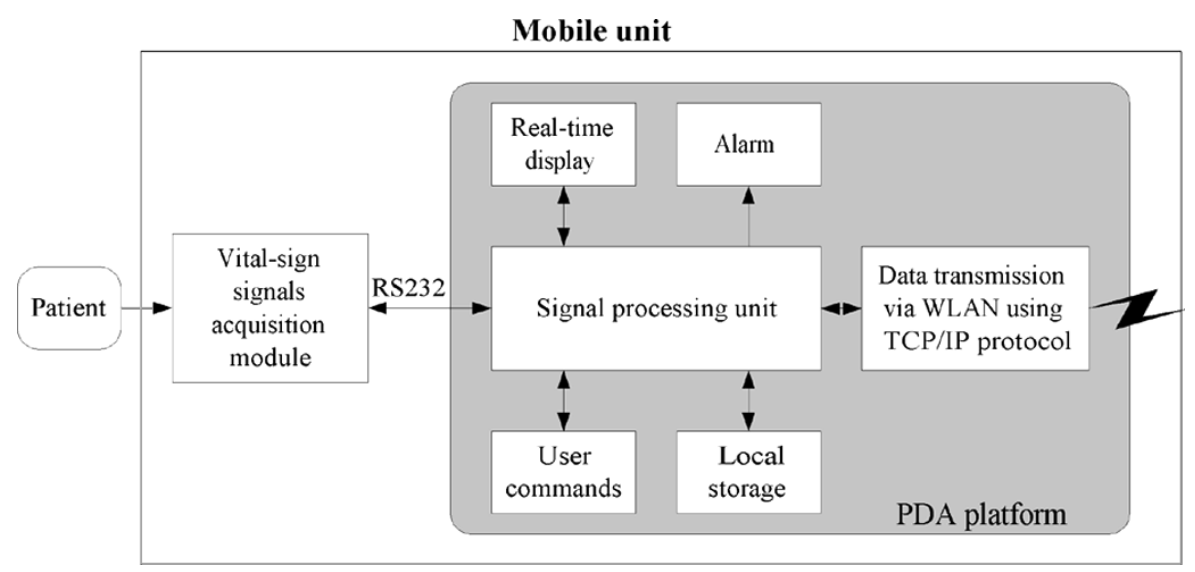

Fig. 2. Design architecture in mobile unit.

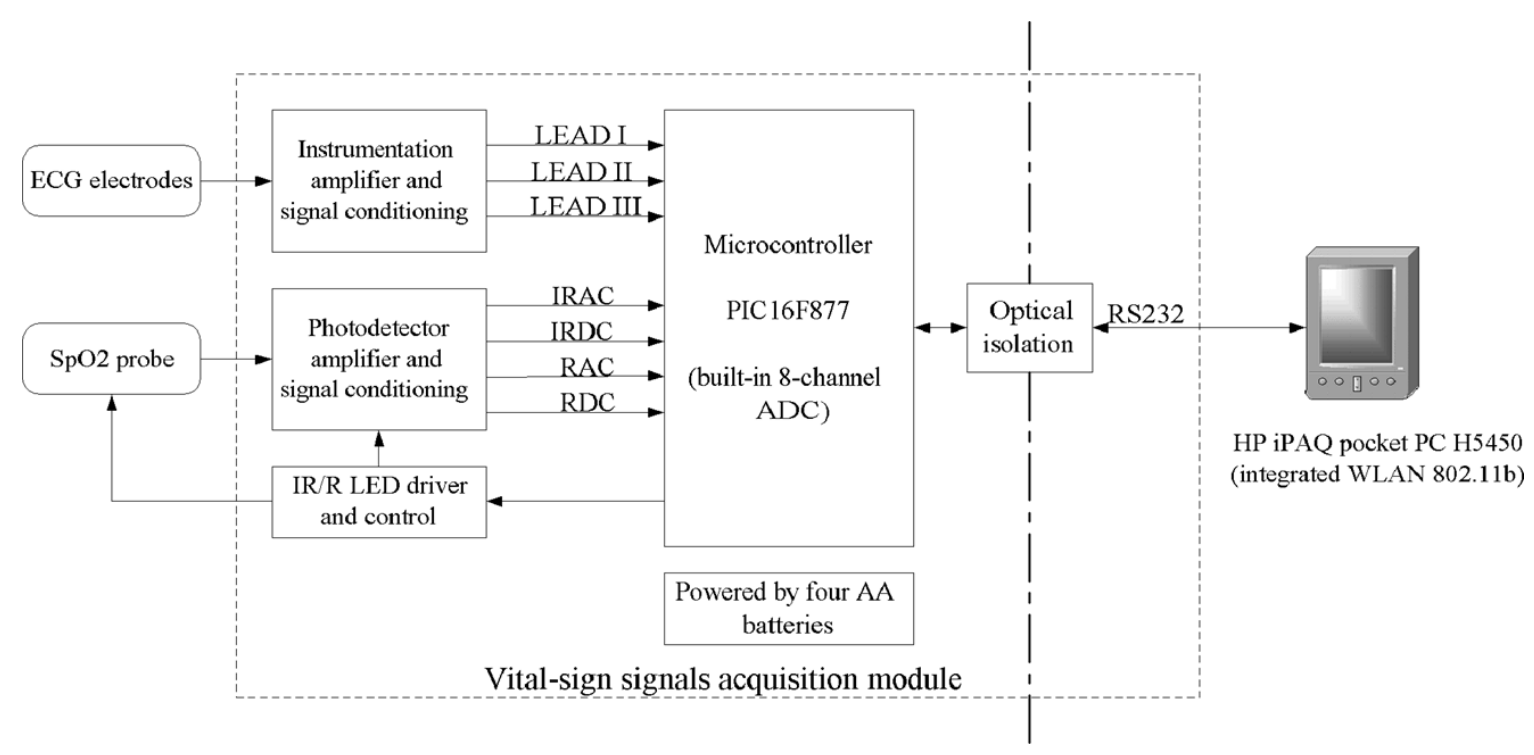

Fig. 3. Block diagram of the designed vital-sign signals acquisition module.

principles of spectrophotometry and optical plethysmography to measure $\mathrm{SpO}_{2}$ [14], and a Nellcor oxygen sensor (DS-100A, finger probe) was used to measure the PPG signals. The signals determined by two light-emitting diodes (infrared and red) are first demultiplexed, then separately amplified, and finally separated into dc and ac components (IRAC, IRDC, RAC, and RDC), which are used to calculate pulse rate and the oxygen saturation in the blood.

The microcontroller digitizes the signals with a sampling frequency of $200 \mathrm{~Hz}$ and transmits the ECG and PPG data to the Pocket PC through the serial port. The baud rate is $115.2 \mathrm{~kb} / \mathrm{s}$. Optical coupling is used in the serial communication to separate the power supply of the signal acquisition module from that of Pocket PC, reducing power interference. Fig. 4 illustrates the mobile unit.

2) Program on the Pocket PC: A system program, developed by Microsoft embedded visual $\mathrm{C}++$, was installed on the Pocket PC to monitor the vital signs. This program records users' information and displays the $\mathrm{HR}, \mathrm{SpO}_{2}, \mathrm{ECG}$, and PPG waveforms sent from the signal acquisition module. Raw data can be stored into the built-in memory of the Pocket PC and transmitted to a remote management unit via the WLAN. In long-term store-and-forward mode, the raw data are stored into the extended secure digital (SD) memory (256 MB) of the Pocket PC. The waveforms are plotted in window with an area of $200 \times 150$ pixels. The amplitude resolution is $0.04 \mathrm{mV} /$ pixel for the three-lead display and $0.0125 \mathrm{mV} /$ pixel for the single-lead display. When the frame displays $4 \mathrm{~s}$ of ECG data, the temporal resolution is $0.02 \mathrm{~s} /$ pixel. Besides, the sound reflecting each heart beat can be pronounced by the speaker of Pocket PC. In addition, this program is installed in the medical staffs' PDAs for receiving and displaying the physiological parameters and waveforms of a remote patient under monitoring through the wireless connection to the management unit.

\section{Management Unit}

Fig. 5 shows the architecture of the management unit. The management unit consists mainly of a fixed personal computer or a laptop, and the management program. The management unit can be set in many spots depending on different applications of telemonitoring. It is normally located in the nurse's station, and provides a user-friendly interface for telemonitoring a patient's vital-sign signals. The management terminal can receive 


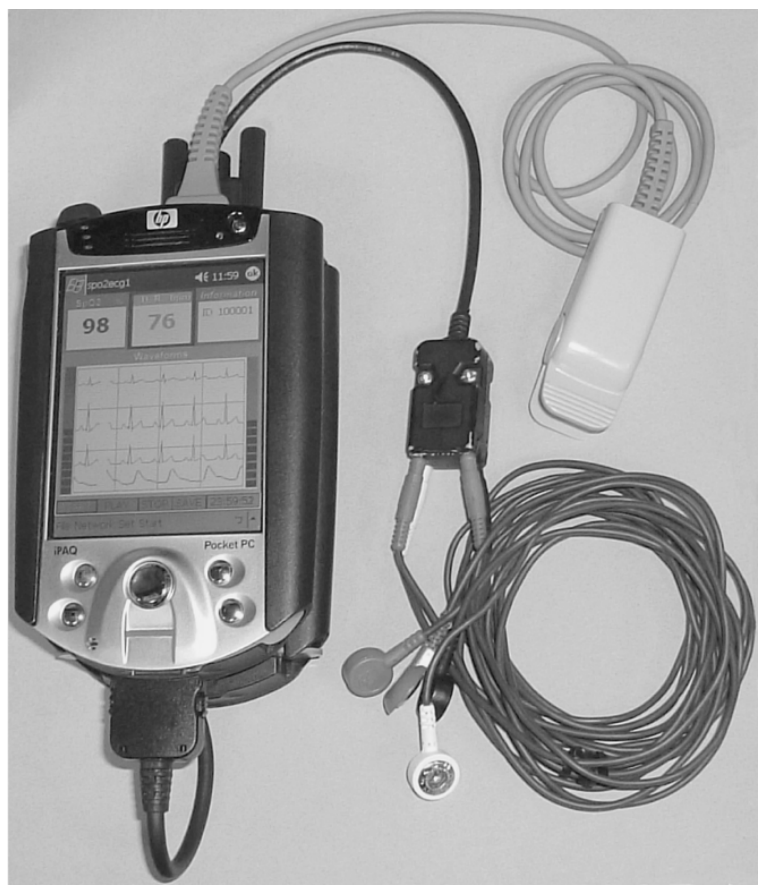

Fig. 4. Picture of the mobile unit.

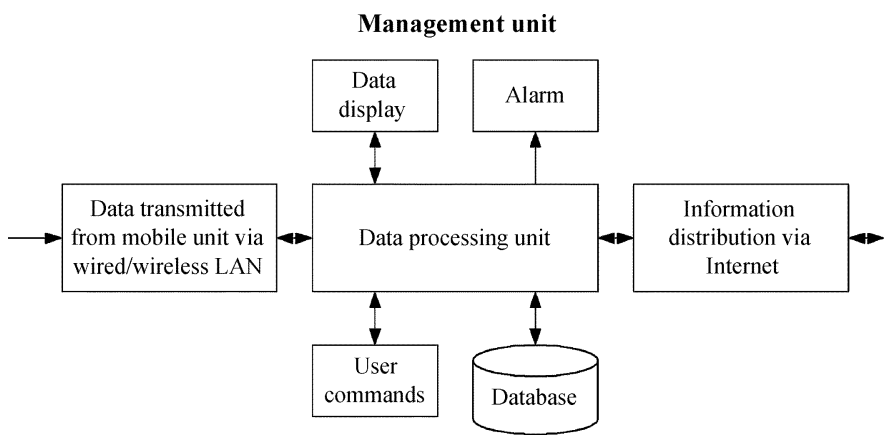

Fig. 5. Architecture of the management unit.

patients' physiological data from the remote mobile units via the WLAN or the Internet.

The management program is implemented on a Windows 2000 platform and developed by the Borland $\mathrm{C}++$ builder. The program receives the data from the mobile unit, displays HR, $\mathrm{SpO}_{2}$, three-lead ECG, and PPG waveforms on the terminal screen, and stores the data in the local database. In this work, a MySQL database system is set up to manage the raw data of ECGs and PPGs, patients' information, and the doctors' diagnosis. The database can also be accessed from authorized terminals through the hospital network and the Internet. Moreover, the vital-sign signal can be delivered in real-time to a mobile platform for sharing data. The waveforms are plotted in a $600 \times 448$ pixels window, which shows $6 \mathrm{~s}$ of ECG data. The default resolutions of amplitude and time are approximately $0.015 \mathrm{mV} /$ pixel and $0.01 \mathrm{~s} /$ pixel, respectively. The program also supports the selection of leads, the replay of waveforms, analysis of raw data, and the scaling of amplitude and time.

Both mobile unit and management unit have an alarm setting window which enables the medical staff to set up the alarm threshold of $\mathrm{SpO}_{2}$ and $\mathrm{HR}$ individually according to the physiological status of the patient. When the recorded vital signs are beyond the preset limits, the mobile unit would trigger an alarm automatically and a warning message window will pop-up on the screen.

\section{Evaluation of System}

The system was evaluated in the following phases.

1) Technical Verification: First, the developed pulse oximeter was calibrated by an index pulse oximeter simulator (Bio-Tek product; $\mathrm{SpO}_{2}$ range: $35 \%-100 \%$; $\mathrm{HR}$ range: 30-250 bpm), whereas the accuracy of the ECG monitor was verified by the medSim 300 Patient Simulator (Dynatech Nevada, Inc.). Then, the functions of the PDA-based pulse oximeter and the ECG monitor, as well as the transmission of data between the mobile unit and the central management unit were tested. Twenty healthy volunteers, including eleven males (with an average age of $29.7 \pm 11$ years old) and nine females (with an average age of $29.6 \pm 10$ years old), were involved in the test. Three-lead ECG signals and PPG signals were acquired simultaneously. All results were recorded locally and were transmitted to the remote central management unit for $5 \mathrm{~min}$ to confirm the quality of the signals and the error rate of data transmission between the two units. Two different probes, one of the designed pulse oximeter and the other of the commercial pulse oximeter BCI-3304 (product of BCI, Inc.) were connected to different fingers of the same volunteer and then operated simultaneously to compare $\mathrm{SpO}_{2}$ and $\mathrm{HR}$ readings over $5 \mathrm{~min}$. During the first minute, the volunteers breathed normally. They were then required to hold their breath for one minute, and then breathe normally until the end of the test.

2) Clinical Test and User Survey: The complete system was demonstrated at National Taiwan University Hospital (NTUH). In the test scenario, each patient was transported from the intensive care unit (ICU) to a radiographic examination room. The mobile unit was placed beside the patient, which enables the medical personnel to observe the patient's physiological condition and check the connection of the electrodes. The mobile unit transmits the patient's vital-sign signals to the management unit via the WLAN, allowing medical staffs to monitor online the patient's data during transport. During the radiographic examination, the mobile unit was placed next to the patient, and a laptop was set up at the control station to monitor the real-time data from the mobile unit.

According to the test scenario, a survey was conducted to elicit the operators' opinions on the wireless PDA-based physiological monitoring system in three areas-1) mobility (size and weight), 2) usability, and 3) performance of the overall system on intrahospital transport. A questionnaire with a five-point Likert scale (from $5=$ completely satisfied to $1=$ completely unsatisfied) was used to rate the performance of the overall system on intrahospital transport. Also, the mobility and usability of the wireless PDA-based monitoring system were compared with the currently used monitoring device (Agilent M3046A) in intrahospital transport at NTUH. The satisfaction of mobility was evaluated in relation to two statements of weight and size and that of usability was estimated by easy 
TABLE I

BATTERY LIFE OF POCKET PC (HP iPAQ H5450)

\begin{tabular}{cccc}
\hline \hline Pocket PC & \multicolumn{3}{c}{ Battery Life (min) } \\
\cline { 2 - 4 } Backlight & Monitoring Only & $\begin{array}{c}\text { Monitoring and } \\
\text { Recording }\end{array}$ & $\begin{array}{c}\text { Monitoring and wireless data } \\
\text { transmission }\end{array}$ \\
\hline Lowest & 194 & 193 & 101 \\
\hline Highest & 125 & 123 & 77 \\
\hline \hline
\end{tabular}

operation and easy monitoring. The outcomes of these four statements were represented as a ten-point scale (from $1=\mathrm{com}$ pletely unsatisfied, to $10=$ completely satisfied). Intrahospital transport scenarios were tested over a one month period in the emergency department. Fifty medical personnel, including 30 nurses and 20 doctors, used the wireless patient monitoring system and answered the questionnaire. The staffs included 14 males and 36 females with ages in the range $23-50$ years old $($ mean $=30 \mathrm{SD}=5.5)$ and with $1-25$ years $($ mean $=6.5$ $\mathrm{SD}=5.0)$ of experience in emergency medical care.

\section{RESULTS}

A prototype of the overall system has been designed and implemented. The mobile unit, which includes a vital-sign signals acquisition module and a wireless PDA, is compact $(13.8 \times 8.4 \times 5 \mathrm{~cm})$ and lightweight $(<500 \mathrm{~g})$. Size of the vital-sign signals acquisition module is small due to the using of surface mount technology devices. The static current consumption of the module is $50 \mathrm{~mA}$, and the dynamic current consumption is $80 \mathrm{~mA}$. This module can continuously acquire biosignals for over $7 \mathrm{~h}$ using $600-\mathrm{mAh}$ AA batteries. The power consumption and continuous in-use time of the Pocket PC (HP iPAQ H5450 including the 1250-mAh lithium-ion polymer battery) are tested. In real-time mode, data are displayed on the Pocket PC's screen and transmitted in real-time to the remote management unit. The Pocket PC can run under its own power for around $70 \mathrm{~min}$ in the worst case. In the store-and-forward mode, the device remained active for approximately $2 \mathrm{~h}$ recording around $20 \mathrm{MB}$ of uncompressed raw data into the $\mathrm{SD}$ memory. The brightness of the backlight and the power of the wireless transmission of the Pocket PC strongly influence the duration of the function of this mobile unit. Table I summarizes the mean battery life in various test scenarios.

Fig. 6 presents the main control and display window of the mobile unit. Both $\mathrm{SpO}_{2}$ and $\mathrm{HR}$ are displayed in the upper area. The middle area plots the ECG and PPG waveforms and also the user can select to display individual waveform. The command buttons are arranged in the lower area.

The technical verification revealed an error in $\mathrm{SpO}_{2}$ of less than $\pm 2 \%$, and an error in HR of less than $\pm 2 \mathrm{bpm}$, between the pulse oximeter that designed herein and the commercial pulse oximeter BCI-3304. Fig. 7 shows a typical 5-min plot of $\mathrm{SpO}_{2}$ and HR. During the initial $60 \mathrm{~s}$, the examinee was breathing normally. The system recorded a stable $\mathrm{SpO}_{2}$ of $96 \%-97 \%$ and an HR of around 70 beats/min. Subsequently, the examinee started to hold his breath. After about $30 \mathrm{~s}$, which stands for the 90th second in the whole process, the concentration of $\mathrm{SpO}_{2}$ started to decline and at the same time the heart beat rate became faster.

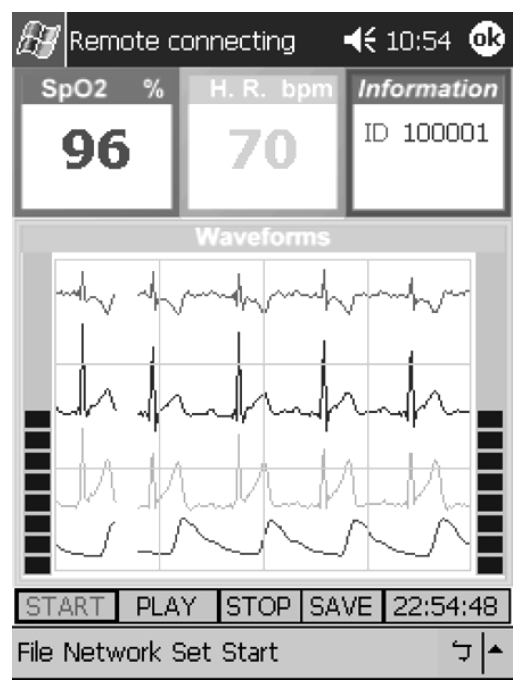

Fig. 6. Main control window and vital-sign signals display of the mobile unit.

After the 120th second, the examinee returned to normal status with the $\mathrm{HR}$ at about 70 beats/min, and his $\mathrm{SpO}_{2}$ also rose from $88 \%$ to $98 \%$.

The wireless data transmission test reveals no error in the real-time vital-sign transmission from the mobile unit to the management unit. The management unit receives the data and adequately displays the patient's information. Fig. 8 presents the test results, the main control, and the display window of the management unit. The data of $\mathrm{SpO}_{2}$ and $\mathrm{HR}$ are shown at the right-hand side and the patient information is shown at the top. The middle area displays the ECG and PPG waveforms, where the user can select to display one specific waveform (ECG Lead I, II, III, or PPG) and scale the time and amplitude. The command buttons are arranged at the right-hand side. The medical staff can open the alarm-setting window to set up the thresholds.

Twenty doctors and thirty nurses who had used the PDA-based monitoring system during patient transport answered the questionnaire. The medical staffs highly rated the overall system (mean $=4.64, \mathrm{SD}=0.53$ ) by a five-point Likert scale (5: completely satisfied, 1: completely unsatisfied) for performance on intrahospital transport. The mobility and usability of the wireless PDA-based system were compared with those of the currently used monitoring system for intrahospital transport at NTUH. The results in Table II indicate that the wireless-PDA model outperforms the traditional monitors in both mobility and usability (at a 95\% confidence level).

\section{DISCUSSION}

A prototype of this PDA-based telemedicine system has been designed and tested. For intrahospital transport of critical patients, experienced senior staffs could monitor the patients online and advise for unexpected condition, and thus, may prevent further deterioration of the patient condition. Under such circumstances, online monitoring or consultation would be helpful. Therefore, the remote monitoring during transport would be beneficial for a better quality care of the patient. We will discuss the following issues that relate to the wireless patient monitoring system. 


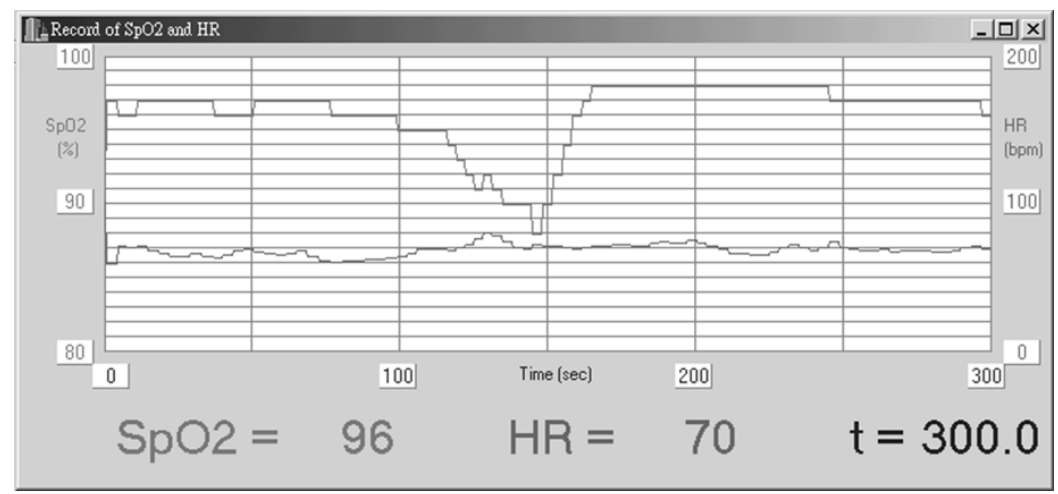

Fig. 7. A 5-min record of $\mathrm{SpO}_{2}$ (upper) and $\mathrm{HR}$ (lower) data.

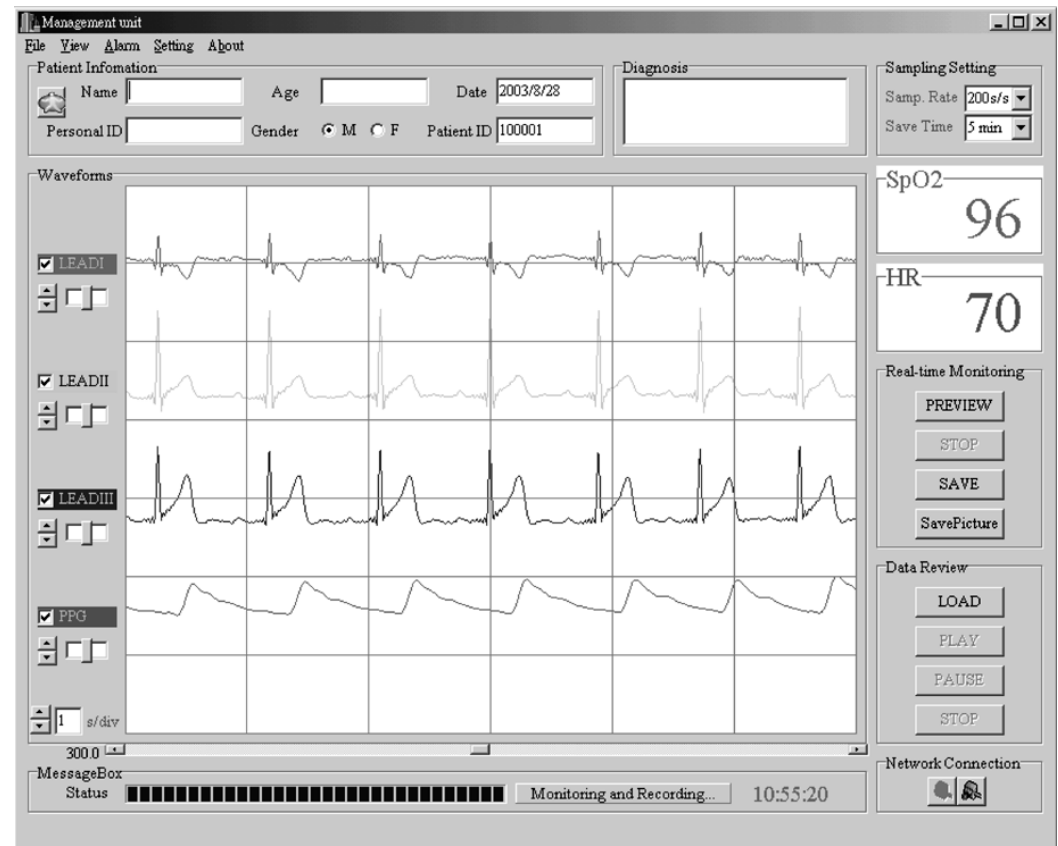

Fig. 8. Main control window and vital-sign signals display of the management unit.

TABLE II

COMPARISON BETWEEN THE WIRELESS-PDA MODEL AND THE CURRENTLY USED MODEL

\begin{tabular}{ccccc}
\hline \hline Score of convenience & \multicolumn{2}{c}{ Wireless-PDA model } & \multicolumn{2}{c}{ Currently used model } \\
& Mean & SD & Mean & SD \\
\hline Weight & 8.8 & 0.9 & 4.7 & 1.5 \\
\hline Size & 8.9 & 0.8 & 4.9 & 1.5 \\
\hline Easy operation & 8.6 & 0.9 & 5.1 & 1.6 \\
\hline Easy monitoring & 8.7 & 1.0 & 5.1 & 1.7 \\
\hline \hline
\end{tabular}

Scoring method: $1=$ completely unsatisfied, to $10=$ completely satisfied. Total 50 medical staffs inquired.

\section{A. Mobility}

The portability of the monitoring device is the key to successful monitoring of mobile patients. The prototype mobile unit is compact and lightweight, which is highly mobile and can be easily carried by medical personnel involved in the transport of a patient. Continuous telemonitoring can be achieved by the wireless technology.

\section{B. Power Consumption}

The power consumed by the monitoring device is also important in the monitoring of mobile patients. According to the test results, the battery life is consumed greatly by using a wireless device, making this wireless PDA-based monitoring device unsuitable for long-term mobile monitoring. However, patient transport and radiographic examination are often completed within $20 \mathrm{~min}$ [15], and the proposed mobile unit is well suited for such applications.

\section{Flexibility}

The telemedicine system based on a WLAN infrastructure is highly flexible and easily constructed anywhere. Flexibility enables this telemedicine system to be extended to several applications. Traditionally, the physiological condition of patients is not accessible during radiographic examination. The proposed system, however, supports continuous monitoring of patients via WLAN connection. The flexibility and high mobility of this system make it useful in numerous applications other than patient transport. For example, the system can be easily established 
in the unit which cares for Severe Acute Respiratory Syndrome patients. The telemonitoring system reduces the need for close contact between health-care personnel and the patients, hence, reducing the chance of infection. Additionally, as WLAN becomes increasingly popular, the system becomes cheaper to establish and maintain. Today, PDA devices are reasonably priced and are becoming increasingly powerful. Therefore, a PDAbased telemedicine system is a cost-effective solution in a range of medical applications and suitable for home care usage.

\section{Usability}

This telemedicine system is user friendly. Both the mobile unit and the management unit provide a graphical user interface. The mobile unit has a hotkey-start that further facilitates its usage. Medical staffs can access patients' information from any location via WLAN which enables more complete medical care to be provided.

Although many devices are available for mobile patient monitoring, those systems are generally bulky and without wireless communication capability and, thus, are not efficient for supporting high mobility or continuous remote monitoring during patient transport. Moreover, the current GSM-based systems have three main drawbacks limiting their widespread use in hospitals: 1) cost effectiveness of the systems and expensive links; 2) limited data transfer rate at $9.6 \mathrm{~kb} / \mathrm{s}$ (currently available); and 3) medical-electronics malfunctions caused by electromagnetic interference of cellular phones. As to the wearable devices, they usually feature extremely low power consumption at the expense of low communication range and bandwidth for long-term monitoring. Although they are easy to carry, the short-range data transmission property restricts their operating range during patient transfer.

In view of this, we integrated our mobile monitoring system with the WLAN technology, which offers a practical and highly flexible means of transmitting data, to meet the need of intrahospital patient care. In addition, the PDA-based monitoring devices also outperform current systems in high-performance data processing, large storage memory, user-friendly interface, upgrading capability, and support of various communication protocols.

Taking the complicated communication environments into consideration, our system can provide store-and-forward mode as well as real-time mode, to assure adequate data transmission. Besides, to further facilitate the remote access, we have also developed a telemonitoring program for the mobile units of the medical staffs. Via remote access with their handheld devices, physicians and other medical staffs can inspect the real-time vital data and assess the changes in patient status so that immediate action can be taken when needed. If sudden, unexpected events happen, emergency procedures can be implemented timely and efficiently.

\section{E. Security}

In the medical world, preventing hackers from illegally accessing private information of patients is very important. In wireless telemedicine applications, because data are transmitted from point to point via radio waves, an individual has the potential to eavesdrop the confidential patient records from wireless network by only a laptop, an antenna, and some specialty software downloaded from the Internet. The $802.11 \mathrm{~b}$ WLAN standard incorporates three mechanisms to provide security for client access to WLAN access points, including the SSID, media access control (MAC) address filtering, and WEP. The SSID identifiers and MAC address filtering could increase security because only authorized client devices can connect through that access point; unfortunately they can be possibly eavesdropped and intercepted by a sophisticated hacker. WEP is designed to provide encryption and authentication as part of the 802.11 standard. Some research teams have disclosed security flaws in 802.11 WEP, including both its encryption algorithm and authentication protocols [16]. To make communication on wireless networks more secure, a robust AES (Rijndael) algorithm has been implemented in the designed program to perform end-to-end encryption, which makes it harder for hackers to intercept and analyze data from network. Using any type of encryption would increase the load of the network. The longer key length used in the encryption algorithm, the more computing power consumed in both mobile and management unit. Therefore, 128-bits key was chosen to use in this AES algorithm.

\section{F. Interference}

WLANs using radio waves have recently gained popularity for installation in hospitals. Because electromagnetic waves transmitted from mobile telephones have been shown to cause interference with medical electronic equipment, prudence would seem necessary when introducing radio-wave communication devices into hospitals. Several studies introduce the tested outcome of the effect of WLAN communication on medical electronic equipment and the effect of electronic equipment on WLAN communication [17], [18]. The results of these studies confirm that very-low intensities generated by WLAN systems are acceptable for use in hospitals, especially in view of the benefits of obtaining real-time access to patients' medical information. In our study, the wireless PDA-based monitoring system have been set up and tested in the ICU and a radiographic examination room. During the test period, none of the related electric equipments malfunctioned. The computer tomography system also did not cause any interference on the wireless monitoring system at distance up to $3 \mathrm{~m}$.

\section{G. Advanced Applications}

The newly approved IEEE $802.11 \mathrm{~g}$ standard specifies data rates of up to $54 \mathrm{Mb} / \mathrm{s}$ in the $2.4-\mathrm{GHz}$ band. It is backwardly compatible with $802.11 \mathrm{~b}$. Increasing bandwidth enables larger amounts of data, such as video images, to be transmitted through the network. Additionally, the multimedia capabilities of PDA are promising; PDAs can be easily equipped with digital cameras, enabling them to be used in various image applications. The integration of multimedia capability and vital sign information on a PDA platform would be helpful in rescue operations.

The designed device can measure ECG and PPG signals simultaneously. Therefore, advanced algorithms, such as for evaluating continuous noninvasive blood pressure by measuring the arrival time of pulse wave, can be implemented in this system [19]. This capability enables many parameters to be determined 
by only a few sensors. The reduction in the number of connections minimizes problems caused during critical patient transport [20]. In addition, Bluetooth might be used to replace the wires of medical surveillance equipment. We plan to use Bluetooth to substitute RS232 in the next design architecture. In the future, this system will incorporate a wireless wide area network, such as GSM or GPRS, to provide an entire emergency health-care system for interhospital and intrahospital patient transport.

\section{CONCLUSION}

A mobile patient monitoring system was designed, developed, and tested. A pulse oximeter was integrated with a threelead ECG monitor on a wireless PDA platform, which provides real-time and store-and-forward modes. The monitor in the new system has a significantly reduced size and weight, and thus, improves the portability of the monitoring device. Besides, WLAN also greatly increases the flexibility and usability for telemonitoring. The clinical evaluation reveals that this mobile patient monitoring system is user-friendly, convenient, and feasible for patient transport.

\section{ACKNOWLEDGMENT}

The authors would like to thank Prof. F.-Y. Lin, the Vice Director of National Taiwan University Hospital, and Dr. M. H.-M. Ma, for their help to coordinate the integration of this study. The authors also thank Dr. N.-K. Chou, Dr. C.-H. Cheng, and Dr. T.-C. Lu, for their help in clinical test. Finally, the authors would like to thank Prof. K.-K. Tiong of the Department of Electrical Engineering, National Taiwan Ocean University, for his invaluable advice on the technical writing.

\section{REFERENCES}

[1] G. De Cosmo, P. Primieri, A. Mascia, E. Gualtieri, V. Bonomo, and A Villani, "Intra-hospital transport of the anaesthetized patient," Eur. J. Anaesth., vol. 10, no. 3, pp. 231-234, May 1993.

[2] Australasian College for Emergency Medicine, "Minimum standards for transport of critically ill paients," Emerg. Med., vol. 15, pp. 202-204, 2003.

[3] C. S. Pattichis, E. Kyriacou, S. Voskarides, M. S. Pattichis, R. Istepanian, and C. N. Schizas, "Wireless telemedicine systems: an overview," IEEE Antennas Propag. Mag., vol. 44, pp. 143-153, Apr. 2002.

[4] S. P. Nelwan, T. B. van Dam, P. Klootwijk, and S. H. Meij, "Ubiquitous mobile access to real-time patient monitoring data," Comput. Cardiology, vol. 29, pp. 557-560, Sept. 2002.

[5] B. Woodward, R. S. H. Istepanian, and C. I. Richards, "Design of a telemedicine system using a mobile telephone," IEEE Trans. Inform. Technol. Biomed., vol. 5, pp. 13-15, Mar. 2001.

[6] K. Hung and Y. T. Zhang, "Implementation of a WAP-based telemedicine system for patient monitoring," IEEE Trans. Inform. Technol. Biomed., vol. 7, pp. 101-107, June 2003.

[7] P. Giovas et al., "Transmission of electrocardiograms from a moving ambulance," J. Telemed. Telecare, vol. 4, no. 1, pp. 5-7, 1998.

[8] S. Pavlopoulos, E. Kyriacou, A. Berler, S. Dembeyiotis, and D. Koutsouris, "A novel emergency telemedicine system based on wireless communication technology-AMBULANCE," IEEE Trans. Inform. Technol. Biomed., vol. 2, pp. 261-267, Dec. 1998.
[9] R. S. H. Istepanian, S. Tachakra, and K. A. Banitsas, "Medical wireless LAN systems (MedLAN). State of the art, challenges, and future directions," in eHealth Conf., Apr. 2001, pp. 43-49.

[10] S. Fischer, T. E. Stewart, S. Mehta, R. Wax, and S. E. Lapinsky, "Handheld computing in medicine," J. Amer. Med. Inform. Assoc., vol. 10, no. 2, pp. 139-149, Mar./Apr. 2003.

[11] K. A. Banitsas, R. S. H. Istepanian, S. Tachakra, and T. J. Owens, "Modeling issues of wireless LANs for accident and emergency departments," in Proc. 23rd Annu. Int. Conf. IEEE EMBS/BMES, vol. 4, Oct. 2001, pp. $3540-3543$.

[12] M. S. Gast, "802.11 Wireless Networks: The Definitive Guide," O’Reilly, 2002.

[13] J. Daemen and V. Rijmen. A Specification for Rijndael, the AES Algorithm. [Online]. Available: http://fp.gladman.plus.com/cryptography_technology/rijndael/aes.spec.311.pdf

[14] J. P. de Kock and L. Tarassenko, "Pulse oximetry: Theoretical and experimental models," Med. Biol. Eng. Comput., vol. 31, pp. 291-300, May 1993.

[15] H. J. Brokakalaki, J. D. Brokakalakis, G. E. Digenis, G. Baltopoulos, L. Anthopoulos, and G. Karvountzis, "Intrahospital transportation: monitoring and risks," Intensive and Cirtical Care Nursing, vol. 12, no. 3, pp. 183-186, June 1996.

[16] N. Borisov, I. Goldberg, and D. Wagner, "Intercepting mobile communications: the insecurity of 802.11," in Proc. 7th Annu. Int. Conf. ACM MOBICOM, July 2001, pp. 180-189.

[17] E. Hanada, Y. Hoshino, H. Oyama, Y. Watanabe, and Y. Nose, "Negligible electromagnetic interaction between medical electronic equipment and $2.4 \mathrm{GHz}$ band wireless LAN," J. Med. Syst., vol. 26, no. 4, pp. 301-308, Aug. 2002.

[18] K. S. Tan and I. Hinberg, "Effects of a wireless LAN system, a telemetry system and electrosurgical devices on medical devices in a hospital environment," Biomed. Instr. Technol., vol. 34, no. 2, pp. 115-118, 2000.

[19] S. O. Heard, A. Lisbon, I. Toth, and R. Ramasubramanian, "An evaluation of a new continuous blood pressure monitoring system in critically ill patients," J. Clin. Anesth., vol. 12, no. 7, pp. 509-518, Nov. 2000.

[20] C. Waydhas, "Intrahospital transport of critically ill patients," Crit. Care, vol. 3, no. 5, pp. R83-R89, 1999.

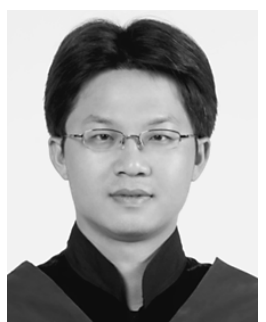

Yuan-Hsiang Lin was born in Yunlin, Taiwan, R.O.C., in 1974. He received the B.S. degree in electronic engineering from National Taiwan University of Science and Technology, Taipei, Taiwan, R.O.C., in 1997. He is now working toward the Ph.D. degree in electrical engineering, National Taiwan University, Taipei, Taiwan, R.O.C.

$\mathrm{He}$ is currently a Teaching Assistant in the Microprocessor Laboratory, Department of Electrical Engineering, National Taiwan University, Taipei, Taiwan, R.O.C., since 2000. His research interests include biomedical instruments, bio-signal processing, and wireless telemedicine.

Mr. Lin has been a Student Member of the IEEE Engineering in Medicine and Biology Society since 2000.

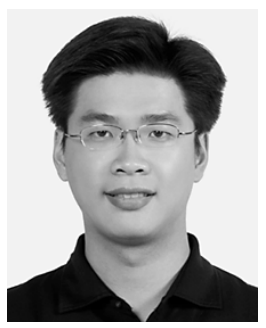

I-Chien Jan received the B.S. degree in physics from National Taiwan University, Taipei, Taiwan, R.O.C., in 1998. He is now working toward the $\mathrm{Ph} . \mathrm{D}$. degree in electrical engineering at National Taiwan University.

His main research areas of interest are biomedical imaging, biophotonics, biophysics, and nanometerscale microscopes. He is now working on the microrheology studies with optical tweezer and differential confocal microscopy. 


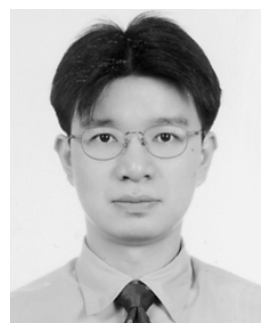

Patrick Chow-In Ko received the M.D. degree from National Taiwan University, College of Medicine, Taipei, Taiwan, R.O.C., in 1995.

$\mathrm{He}$ is currently an Attending Physician in the Department of Emergency Medicine, National Taiwan University Hospital, Taiwan, R.O.C.; an Instructor for the Emergency Medical Service of the National Fire Department; and a Medical Instructor for the Urban Search and Rescue Task Force of Taipei City. His research activities have focused on emergency medical services, resuscitation, and critical care transport. His work has been published in several international conference proceedings and scientific journals of emergency medicine.

Dr. Ko was certificated as a Physician in Internal Medicine, in 1998, and a Specialist in Emergency Medicine, in 2000.

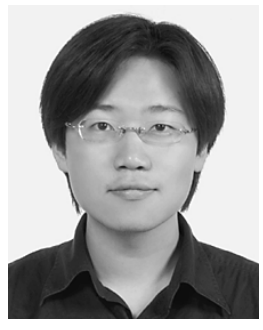

Yen-Yu Chen received the degree in electrical engineering from National Taiwan University, Taipei, Taiwan, R.O.C., in 1999. He is currently working toward the Ph.D. degree at the National Taiwan University.

After graduating, he joined the Laboratory of Bioptics, National Taiwan University, Taipei, Taiwan, R.O.C., and began his study on laser Doppler flowmetry. His main areas of interest are light scattering spectroscopy, signal processing, and fluid dynamics analysis. He is now working on the functional property analysis of micro-fluids using light scattering spectroscopy. Mr. Chen has been a Student Member of the IEEE Engineering in Medicine and Biology Society since 2000.

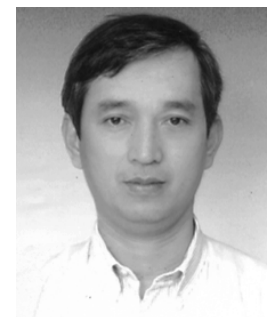

Jau-Min Wong received the M.D. and Ph.D. degrees from National Taiwan University, Taipei, Taiwan, R.O.C., in 1978 and 1994, respectively.

He was a Visiting Research Fellow at Dana Faber Cancer Institute, Harvard Medical School, Boston, MA between 1987 and 1989 . He is currently an Associate Professor in the School of Medicine, Institute of Biomedical Engineering and Institute of Preventive Medicine, National Taiwan University. $\mathrm{He}$ is also currently a Gastroenterology Specialist in the Department of Internal Medicine, National Taiwan University Hospital, Taipei, Taiwan. His research activities have focused on knowledge development in biomedical database.

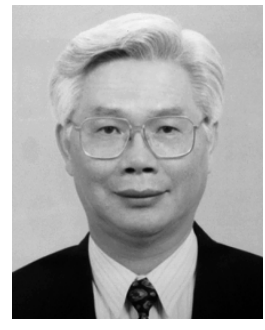

Gwo-Jen Jan was born in Taiwan, R.O.C., in 1946. He received the B.S. degree from the Chung-Yuan Christian Science and Engineering College, in 1968, the M.S. degree from National Taiwan University, in 1970, and the Ph.D. degree from the City University of New York, New York, in 1982, all in physics.

Since 1986, he has been a Professor in the Department of Electrical Engineering and also has been working at the Graduate Institute of Electrooptic Engineering, National Taiwan University, Taipei, Taiwan, R.O.C., since 1994. He is currently involved in research on the optical characterization of semiconductor materials and devices. His research is also involved in the design and development of the biophotonic and biomedical electronic instrumentation systems. 